\title{
Intégrer une communauté dans une autre : Barack Obama et Amazing Grace
}

Integrating One Community into Another: Barack Obama and Amazing Grace

\section{Fiona Rossette}

\section{(2) OpenEdition}

12 Journals

\section{Édition électronique}

URL : http://journals.openedition.org/aad/2799

DOI : $10.4000 /$ aad. 2799

ISSN : 1565-8961

Éditeur

Université de Tel-Aviv

Référence électronique

Fiona Rossette, "Intégrer une communauté dans une autre : Barack Obama et Amazing Grace », Argumentation et Analyse du Discours [En ligne], 21 | 2018, mis en ligne le 15 octobre 2018, consulté le 23 septembre 2019. URL : http://journals.openedition.org/aad/2799 ; DOI : 10.4000/aad.2799

Ce document a été généré automatiquement le 23 septembre 2019.

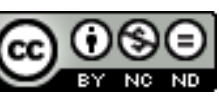

Argumentation \& analyse du discours est mis à disposition selon les termes de la licence Creative Commons Attribution - Pas d'Utilisation Commerciale - Pas de Modification 4.0 International. 


\section{Intégrer une communauté dans une autre : Barack Obama et Amazing Grace}

Integrating One Community into Another: Barack Obama and Amazing Grace

Fiona Rossette

\section{Introduction}

1 En juin 2015, Barack Obama prend la parole pour faire l'éloge funèbre du pasteur noir Clementa Pickney et de ses huit fidèles assassinés lors d'une séance de prière dans leur église à Charleston. Qualifié d'historique - le New York Times a intitulé son article «L'éloge funèbre prononcé par Obama, qui trouva sa place dans l'histoire ${ }^{1}-$, le discours attira surtout l'attention pour sa péroraison, dans laquelle le Président chanta plusieurs vers de Amazing Grace en entraînant son auditoire.

2 Cet éloge va nous permettre de réfléchir aux ressorts de ce type de discours épidictique. Sans doute un grand nombre de travaux de rhétorique sur le discours politique américain étudient-ils l'influence du religieux sur le politique. Le recours au religieux y est alors considéré comme un procédé parmi d'autres pour susciter l'adhésion à des positions politiques. L'intérêt du discours de Charleston est cependant qu'il parvient à dépasser la distinction entre discours religieux et discours politique dans une situation de parole qui relève de manière exemplaire de l'épidictique, lequel vise à un « effet de communion» (Perelman et Olbrechts-Tyteca $1970: 67)^{2}$.

3 Je m'appuierai en particulier sur les concepts de «Scène rhétorique " (Maingueneau 2016) et de "particitation» (Maingueneau 2004) empruntés aux problématiques de l'énonciation (que je définis plus loin). Ces deux concepts permettent de mieux comprendre la manière dont Obama réussit non seulement à susciter un moment de communion entre orateur et auditoire mais également à intégrer imaginairement une communauté, les Afro-Américains, dans une autre, le peuple américain. L'efficacité de 
la particitation est renforcée ici par le fait qu'elle s'appuie sur un ensemble de rituels propres à la pratique religieuse afro-américaine, qui implique un fort investissement corporel.

4 Après avoir présenté les enjeux de ce discours, j'analyserai la mise en œuvre de la Scène rhétorique, puis j'aborderai la question de la particitation associée au chant.

\section{Un discours pas comme les autres}

\subsection{Le contexte}

5 Comme le rapporte le journal Le Monde (Corine Lesnes, 26/6/15), le passage de la parole au chant dans le discours de Charleston surprit d'abord l'auditoire, avant de susciter un «moment de communion»:

Dans le grand hall de l'université de Charleston, les 5000 participants massés dans les gradins n'ont pas compris tout de suite l'invitation à ce moment de communion. Derrière l'orateur, les religieux en soutane violette de la congrégation méthodiste ont souri, presque amusés, devant pareille audace. L'organiste a mis un moment à décider quelle contenance adopter face à cette entorse au protocole, et il lui a fallu un bon moment encore pour trouver la note juste. Mais Barack Obama ne s'est pas contenté des premières mesures. Il a poursuivi, sa voix s'est élevée, généreuse, et l'assistance, debout, s'est laissé entraîner par la ferveur : «J'étais perdu mais je suis retrouvé. J'étais aveugle, maintenant je vois » [...].

En effet, le chant appelle à la participation de l'auditoire : Obama entonne l'hymne, a capella, puis des membres de l'auditoire se joignent à lui, suivis par l'organiste, pour créer une véritable zone de partage où les différences religieuses, ethniques, politiques entre les membres de l'auditoire sont dépassées par l'appartenance à une même communauté.

\subsection{Une double orientation : politique et religieuse}

7 La pratique politique associe régulièrement meeting politique et chant - on pense notamment aux meetings en France qui se terminent par la Marseillaise ou par l'Internationale, ou aux meetings américains auxquels on invite des vedettes à venir chanter. Mais il n'est pas commun de voir un homme politique chanter avant d'avoir achevé son discours. Par ailleurs, le cas d'obama est particulier car la situation de communication et la stratégie rhétorique qu'il développe l'amènent à ne pas se poser seulement en homme politique. Pour le journal Le Monde, lors du discours de Charleston, il a «surtout trouvé les accents d'un pasteur, celui de la nation. Et un pasteur noir». Le journal repère ce double statut de l'orateur en jouant sur la polysémie de "pasteur ", qui renvoie à la figure du clerc et en même temps à celle du guide de la nation. On retrouve le même propos, pour l'essentiel, dans le Financial Times, pour qui le discours se situe « à mi-chemin entre discours présidentiel et sermon »3.

8 Certes, cette double orientation s'explique également par le contexte : même si la scène se passe dans un amphithéâtre universitaire, Obama est entouré des membres de l'Église Africaine Méthodiste Episcopale («AME Church») rassemblés sur le podium, après une fusillade qui eut lieu au sein de l'une de leurs églises et dont la première victime était à la fois pasteur et sénateur. Cependant, rien n'obligeait le Président à se conformer à la pratique de la prédication afro-américaine qui consiste à passer de la 
parole au chant. De même, rien ne l'obligeait à mettre l'accent sur son action politique en abordant la question des lois en matière de contrôle des armes à feu. C'est ce qui distingue l'éloge de Charleston d'un autre éloge funèbre, prononcé par le Président quatre ans plus tôt, en 2011, cette fois à Tucson, en l'honneur de six victimes tuées lors d'une réunion qui rassemblait une parlementaire démocrate et ses administrés. Dans le discours de Tucson, considéré à l'époque comme l'un des meilleurs prononcés jusque-là par le Président, ce dernier se garde bien d'introduire une note religieuse aussi forte, et encore moins de s'aventurer sur un quelconque terrain politique.

La double orientation du discours de Charleston va de pair avec un dédoublement de l'auditoire qui est ciblé. En effet, le Président s'adresse aux proches des défunts et aux membres de l'Église, qui font tous deux partie de la communauté noire, et en même temps à l'ensemble du peuple américain. À ce propos, soulignons qu'Obama ne choisit pas n'importe quel chant: Amazing Grace n'est pas un negro spiritual propre à la communauté noire, mais l'hymne religieux le plus célèbre de la culture anglo-saxonne, écrit par un clergyman anglais ${ }^{4}$. Ce choix permet de satisfaire simultanément deux exigences divergentes : manifester une solidarité - voire une appartenance - avec la communauté noire sans cesser d'être le Président de tous les Américains.

\subsection{Communion et dispositifs énonciatifs}

Le rôle de l'auditoire est d'autant plus important que le discours suscite un véritable sentiment de communion, composant essentiel de l'épidictique. Cette communion est étroitement liée aux pratiques religieuses afro-américaines. Les liens intertextuels avec les discours de Martin Luther King sont évidents. Comme le soulignent des travaux américains de rhétorique et de cultural studies, la participation de l'auditoire, tout comme l'importance de la musique et du rythme, sont constitutives de l'art oratoire afro-américain qui vise ainsi à susciter un sentiment de communion (Alkebulan 2003). On insiste notamment sur le rôle du chant qui unifie l'auditoire (Rosenberg 1971) et sur la pratique interactive qualifiée de call-response (Harrison et Harrison 1993) par laquelle l'orateur apostrophe l'auditoire, qui répond par des formules ritualisées. Ce sont là des composants de la "nouvelle homilétique», qui n'est plus fondée sur l'autorité de l'orateur mais sur un "dialogue» co-construit avec l'auditoire, associé à une performance qui engage tout le corps (Craddock 2001 ; Wesley Allen 2010). Ces pratiques fondent une "fonction homilétique " - "sermonic function" (CallowayThomas et Lucaites 1993 : 6) - qui, depuis les années soixante, s'étendrait au domaine du discours public profane : ce dernier s'avère alors « religieux » dans le sens de religio ("s'unir »), où le discours agit comme une force qui rassemble ses participants.

11 Mais si désormais l'auditoire participe à une co-construction du discours, orateur et auditoire sont-ils pour autant au même niveau ? S'il se crée un « dialogue », celui-ci estil comparable au phénomène de "conversationnalisation » qui s'observe désormais dans le discours public (Fairclough 1994, Crystal 2001)? En fait, les ressorts linguistiques de cette co-construction ont été peu étudiés, tout comme la communion qu'elle engendrerait - à l'exception des travaux récents de Malmström (2016), qui étudie les marqueurs de subjectivité sur un corpus de sermons anglo-saxons.

Des concepts issus de l'analyse du discours française peuvent sur ce point s'avérer utiles. La Scène rhétorique (Maingueneau 2016) renvoie à un dispositif énonciatif spécifique où le locuteur se décale vers un autre plan par rapport à celui occupé par le 
destinataire, pour devenir un « surlocuteur » qui prend « de la hauteur » afin d'étendre le champ de ses destinataires - ce qui lui permet ainsi de consolider la communauté. Quant au concept de "particitation » (Maingueneau 2004), il met en lumière le procédé qui consiste à citer des paroles connues d'une communauté - ici, en l'occurrence, les vers d'Amazing Grace - afin de renforcer le lien entre ses membres. En se faisant ainsi le porte-parole de cet héritage commun, le locuteur, s'associe à ses destinataire(s) dans le partage d'un même « hyperénonciateur $»^{5}$.

\section{La Scène rhétorique}

\subsection{L'asymétrie constitutive de l'art oratoire}

Contrairement à l'oral conversationnel, l'art oratoire associe oralité et parole monologale selon un dispositif que l'on peut qualifier d'«oral élaboré» (Rossette 2017a). Il s'agit d'un dispositif fondé sur une asymétrie constitutive, où un seul locuteur s'adresse à des destinataires multiples (l'auditoire). L'altérité associée au statut du locuteur est matérialisée par le podium : physiquement, le locuteur occupe un espace bien distinct, situé généralement en hauteur. À la lumière des notions de "partition " et de "frontière » développées dans le cadre de l'analyse des genres théâtraux (" performance genres" ou "platform skills») par Goffman (1981: 166), cette relation asymétrique marque une "distance» ou un «seuil discursif» qu'il convient de combler. En effet, si cette distance confère l'autorité nécessaire au locuteur pour prendre la parole devant la foule, elle constitue en même temps un espace qu'il faut combler puisque le locuteur doit parvenir à toucher son auditoire, à créer un lien avec lui.

\subsection{Un orateur-surlocuteur}

Afin de parvenir à créer un tel lien, une tendance des pratiques actuelles consiste à cultiver, à l'intérieur du dispositif monologal, des formes linguistiques propres au dispositif dialogal de l'oral conversationnel. Lorsqu'elles sont déployées en contexte monologal, ces formes apportent une couleur « dialogique » et deviennent des « formes dialogiques ». Regroupant des formes assez hétérogènes, cette catégorie participe à l'effet de "conversationnalisation » qui s'introduit désormais dans le discours public (Fairclough 1994, Crystal 2001), et qui s'observe dans les discours politiques en langue anglaise, qu'ils soient américains (Wilson 2015 : 201) ou britanniques (Debras et L'Hôte 2015 : 189), ainsi que dans d'autres types de discours ${ }^{6}$. Dans ce cas, on parle par exemple de la volonté de créer une proximité, ou "une relation intime malgré la distance » (Horton et Wohl 1956), ou d'instaurer une « dimension collaborative» (Capone 2010).

Or, le dispositif de la Scène rhétorique va à l'encontre de cette tendance actuelle. Le locuteur y joue de la distance constitutive qui le sépare de l'auditoire, pour assumer un statut élevé, qui rappelle l'image noble à laquelle est associée la rhétorique antique. Telle qu'elle est définie par Maingueneau $(2016)^{7}$, la Scène rhétorique se distingue de la conversation par le fait que le locuteur "prend de la hauteur» pour devenir un «surlocuteur ». Ce statut « décalé » lui permet d'étendre le champ de ses destinataires, de s'adresser à un ensemble plus vaste, associé à un "surdestinataire». Ce dernier terme est adapté de Bakhtine (1984: 337) pour nommer «celui qui transcende 
l'interaction verbale", avec la différence qu'il désigne ici une entité qui inclut le destinataire immédiat, le public qui assiste en direct à la scène.

Ainsi, par son dispositif énonciatif même, dont les participants dépassent l'ici-etmaintenant de la prise de parole, la Scène rhétorique construit la communauté qui fonde l'épidictique. Cette communauté, englobante, constituée par l'ensemble des participants physiquement présents et absents, est fondée sur des valeurs communes qui sont en général explicitées dans le discours. Ce dispositif est représenté par la figure 1. On peut considérer que le terme d'«orateur» suffit pour désigner ce surlocuteur, dont le podium symbolise la position élevée.

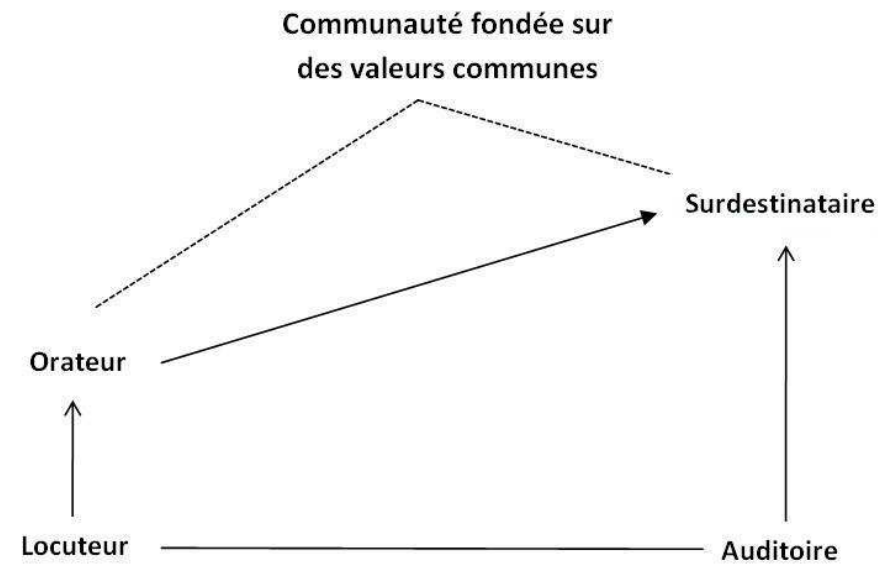

Figure 1 : Scène rhétorique et réseau de participants

Figure 1 : Scène rhétorique et réseau de participants Charleston et qui convertit, à travers l'acte discursif, un auditoire en communauté. Plus
précisément, Obama s'adresse à la fois à la communauté religieuse, l'Église AME, et donc au peuple noir, et à tout le pays. Il parle «à travers » les premiers (l'auditoire physiquement présent, dont il s'entoure), afin d'atteindre les seconds, pour rassembler le tout au sein d'une même entité que constitue le surdestinataire ${ }^{8}$.

Le champ des destinataires va s'élargir peu à peu, au cours du discours, à partir du cercle des intimes :

1. To Jennifer, his beloved wife; to Eliana and Malana, his beautiful, wonderful daughters; to the Mother Emanuel family and the people of Charleston, the people of South Carolina.

Énoncée sous forme averbale, structure marquée en anglais, cette énumération prend la couleur performative d'une dédicace. Plus loin, le Président se tourne vers la communauté de l'Église AME, composée de "nos frères et sœurs » :

2. Clem was often asked why he chose to be a pastor and a public servant. But the person who asked probably didn't know the history of the AME church. As our brothers and sisters in the AME church know, we don't make those distinctions. 
Ainsi, Obama s'identifie à cette communauté, étape nécessaire pour l'orateur, qui intègre, lui aussi, la communauté englobante ${ }^{9}$. A ce titre, la mise en scène est révélatrice : Obama prend la parole face au cercueil du défunt, entouré des membres de l'Église AME, dont la couleur pourpre s'affiche sur le pupitre, sur les chasubles... et jusque, nous semble-t-il, dans la cravate portée par le Président. De façon significative, cette mise en scène offre un contraste saisissant avec celle adoptée quatre ans auparavant pour l'éloge de Tucson où, isolé devant un paravent bleu et derrière un pupitre qui affiche le sceau présidentiel, Obama parle uniquement en tant que Président ${ }^{10}$.

21 La mise en scène de Charleston, tout comme les références interpersonnelles, renvoient à un orateur qui négocie entre la communauté des noirs et celle de la nation, afin d'intégrer la première dans la seconde. Le passage suivant comporte un jeu intéressant où le possessif de la première personne du pluriel, our, renvoie tantôt à la nation, tantôt à la communauté des noirs américains :

3. To the families of the fallen, the nation shares in your grief. Our pain cuts that much deeper because it happened in a church. The church is and always has been the center of African-American life, a place to call our own in a too often hostile world, a sanctuary from so many hardships.

Par la juxtaposition des énoncés, on met sur le même plan "the nation " et "our pain ", et on en conclut qu'Obama parle ici au nom de la nation. Cependant, dans l'énoncé qui suit, on ne sait pas si our («a place to call our own») renvoie à la communauté noire ou à tout le peuple américain. Il s'agit d'un passage clé à plus d'un titre car Obama introduit ici l'édifice religieux, endroit sacré où a eu lieu le massacre et qui, de manière générale, a joué un rôle important dans l'histoire du peuple noir américain. Il parle de l'« Église noire » non seulement comme sanctuaire mais comme lieu de vie, déployant à nouveau la première personne du pluriel :

4. Over the course of centuries, black churches served as "hush harbors" where slaves could worship in safety; praise houses where their free descendants could gather and shout hallelujah; rest stops for the weary along the Underground Railroad; bunkers for the foot soldiers of the Civil Rights Movement. They have been, and continue to be, community centers where we organize for jobs and justice; places of scholarship and network; places where children are loved and fed and kept out of harm's way, and told that they are beautiful and smart and taught that they matter. That's what happens in church.

That's what the black church means. Our beating heart. The place where our dignity as a people is inviolate [...] (c'est nous qui soulignons).

Par la suite, il élargit le propos à l'ensemble du peuple américain. "Church», sans qualificatif, prend le pas sur «black church », et le glissement se fait précisément par l'explicitation des valeurs qui fondent la communauté englobante (« human rights», « human dignity », « liberty », « justice ») :

5. A sacred place, this church. Not just for blacks, not just for Christians, but for every American who cares about the steady expansion of human rights and human dignity in this country; a foundation stone for liberty and justice for all. That's what the church meant.

Ce rappel historique sert à renforcer le statut de l'orateur qui adopte une vision surplombante. Il se réfère à l'histoire pour consolider la communauté, qui ne se limite pas au moment présent, et comprend non seulement les vivants mais aussi les morts. En même temps, l'orateur légitime son propre discours par le fait qu'il s'approprie une généalogie, acte décisif pour devenir surlocuteur (Maingueneau 2016). 

rhétorique se caractérise par une mise en spectacle, une attention particulière au signifiant converti en objet esthétique. La mise en spectacle est à lier aux valeurs morales qui sous-tendent la communauté :

Dans la Scène rhétorique, cette mise en spectacle est à prendre au sens fort : elle est mise en spectacle non seulement de la relation entre les interactants, mais de la parole elle-même, qui se doit d'être exemplaire esthétiquement et éthiquement. Idéalement, cette parole qui en ces circonstances énonce ce qu'il faut énoncer, et de la manière qu'il faut, le Beau et le Bien se fondent. (Maingueneau 2016 : 26)

Cette langue qui se veut belle, rappelant l'apparat et le spectacle associés traditionnellement - et de façon quelque peu négative ${ }^{11}$ - à l'épidictique, va à l'encontre de la tendance actuelle qui consiste à privilégier des formes dialogiques afin de créer un effet de « conversationnalisation ».

Sur ce point l'éloge de Charleston contraste avec l'éloge prononcé par Obama à Tucson. Dans ce dernier se déploient des formes dialogiques comme les interrogatives directes et les marqueurs discursifs (ex. you see, yes), ainsi qu'un grand nombre de coordonnants (and, but), principaux connecteurs de l'oral conversationnel en anglais. Or, le discours de Charleston présente une textualité toute autre, dont la cohésion est assurée non pas par des connecteurs mais par le recours massif aux figures rhétoriques de répétition et aux énoncés averbaux. Ces deux catégories de constructions, absentes de l'anglais conversationnel, participent ici à la mise en spectacle de la parole. Elles produisent une syntaxe extrêmement épurée, à l'image du Gettysburg Address (1863) d'Abraham Lincoln, l'éloge funèbre le plus célèbre de la culture anglo-saxonne, qui établit, en matière de style oratoire en langue anglaise, une nouvelle norme moderne : a new, lean language (Wills 1992). 
31 Est omniprésente dans le discours de Charleston la principale figure rhétorique des discours politiques, à savoir l'anaphore : on y compte dix-neuf séries rassemblant chaque fois deux ou trois énoncés ${ }^{12}$. L'épiphore (qui place le segment répété en position finale d'énoncé) joue également un rôle important, marquant notamment, comme on observera plus loin, la péroraison («Clementa Pinckney found that grace. Cynthia Hurd found that grace.... ") ${ }^{13}$. Obama fait également usage d'accumulations (synathroesmus), notion dont on se sert en général lorsqu'il y a au moins quatre répétitions de la même structure, à l'instar du passage suivant, qui contient par ailleurs un rare exemple dans ce discours de la première personne du singulier $I$ :

7. The first thing I noticed was his [Reverend Pinckney's] graciousness, his smile, his reassuring baritone, his deceptive sense of humor [...]

Dans l'extrait suivant, l'effet de la structure ternaire du premier énoncé s'ajoute à l'effet d'amplification produite par l'accumulation (dernier énoncé) :

8. He was in the pulpit by 13 , pastor by 18 , public servant by 23 . He did not exhibit any of the cockiness of youth, nor youth's insecurities; instead, he set an example worthy of his position, wise beyond his years, in his speech, in his conduct, in his love, faith, and purity.

33 Structure ternaire et accumulation sont mises en relief ici par la voix, dont le débit s'accélère et qui devient de plus en plus forte. En effet, les figures informent des variations rythmiques et vocales particulièrement frappantes, composant clé de la mise en spectacle d'un discours destiné à être prononcé à voix haute, théâtralisé - à l'instar des discours de Martin Luther King, pour lesquels on a souligné l'importance du rythme (Queen 1992, Calloway-Thomas et Lucaites 1993, Shih et Kochanski 2001), et des figures rhétoriques (Lischer 1995), procédés caractéristiques de la prédication afro-américaine (Rosenberg 1971). Nous considérons, comme Magri-Mourges (2015) qui étudie la répétition, que l'apport des figures de rhétorique se situe moins sur le plan dénotatif que sur le plan interpersonnel et illocutoire - et tout particulièrement quand il s'agit de susciter un sentiment de la communion.

34 A côté de ces figures rhétoriques, les énoncés averbaux participent également de la mise en spectacle de la parole. Cette structure, particulièrement marquée en anglais, est employée depuis peu de temps dans le discours public (Rossette 2017a). Un quart des énoncés du discours de Charleston (49 sur 180) prend la forme d'un énoncé averbal ${ }^{14}$, construction qui est présente dès les premières lignes du discours, où elle s'associe à la figure de l'anaphore («A man who... ») :

9. We are here today to remember a man of God who lived by faith. A man who believed in things not seen. A man who believed there were better days ahead, off in the distance. A man of service who persevered, knowing full well he would not receive all those things he was promised, because he believed his efforts would deliver a better life for those who followed.

C'est également par le biais de l'énoncé averbal qu'est mis en relief le thème de la grâce lorsqu'il est introduit pour la première fois dans le discours, avant de faire l'objet d'un long développement qui préfigure le choix du chant Amazing Grace :

10. According to the Christian tradition, grace is not earned. Grace is not merited. It's not something we deserve. Rather, grace is the free and benevolent favor of God as manifested in the salvation of sinners and the bestowal of blessings. Grace.

À l'instar du dernier énoncé de ce passage, l'énoncé averbal joue un rôle important en termes de focalisation. De façon paradoxale, il ne va pas de pair avec un refus du verbe : il dénote souvent au contraire une forte valeur déclamatoire, voire performative. C'est 
ce qui ressort d'un autre passage, composé uniquement d'énoncés averbaux, où sont énumérés les noms des victimes de la tuerie. Ceux-ci sont suivis chaque fois d'une pause (indiquée par une barre oblique) qui renforce l'effet de focalisation. Dépourvu de verbe de prédication, chaque nom propre - un signifiant " mis à nu » mais en même temps resémantisé - permet de rendre hommage de façon autonome à chaque victime et en même temps de réaliser une sorte de déconnexion, pour donner un sentiment d'intemporalité, à la manière d'une épitaphe :

11.Cynthia Hurd. / Susie Jackson. / Ethel Lance. / DePayne Middleton-Doctor / [...].

/ Good people. / Decent people. / God-fearing people. / People so full of life and so full of kindness. / People who ran the race, who persevered. / People of great faith.

/

Si les noms des victimes occupent la première moitié de ce passage, dans la deuxième moitié figure la liste de leurs vertus (Good people. / Decent people...), qui sont élevées au rang des valeurs de la communauté ici célébrée.

On observe à quel point la mise en spectacle de la parole est au service de la construction de la communauté englobante, et comment s'articulent dans la Scène rhétorique réseau interpersonnel et mise en spectacle. C'est à l'intérieur d'un tel dispositif qu'intervient l'auditoire - participation qui se fait sous le contrôle d'un locuteur devenu surlocuteur, à la fois sur un mode ritualisé emprunté à la pratique religieuse et sur le mode de la " particitation ».

\section{Participation, incorporation et particitation}

\subsection{Une participation ritualisée qui engage tout le corps}

Lorsqu'Obama se met à chanter, l'assemblée réagit immédiatement: les membres de l'Église présents sur le podium se lèvent, puis chantent. Une bonne partie de l'auditoire dans la salle prend vite le pas, suivie par l'organiste :

12. [Chanté:] Amazing [rires de l'auditoire] grace, [des membres de l'Église se lèvent] how sweet the sound; [l'auditoire se met à chanter] that saved [début de l'orgue] a wretch like me; I once was lost, but now I'm found; was blind but now I see [applaudissements]

Ce passage exemplifie différents procédés de participation typique des rites de l'Église afro-américaine : applaudissements, rires, gestes, accompagnement musical. L'adoption du même comportement qui engage tout le corps renforce l'intégration au sein d'une même communauté (Craddock 2001, Wesley Allen 2010). Véritable performance qui amplifie la mise en spectacle de la parole, elle suscite également une « incorporation », l'intégration au sein de la communauté sur le plan sensoriel.

41 Cette participation s'est exprimée dès le début du discours. Alors que le Président prend place sur le podium, l'auditoire manifeste sa présence par des applaudissements de même que par une autre forme de participation typique du contexte religieux afroaméricain de type interjectif, dont les réponses ritualisées call-response :

13. Giving all praise and honor to God [applaud. + "yeah!"]. The Bible calls us to hope. To persevere ["aha!" "yeah!"] and have faith in things not seen ["aha!" "praise!"]. "They were still living by faith when they died," [“yeah!"] Scripture tells us. "They did not receive the things promised; they only saw them and welcomed them from a distance, admitting that they were foreigners and strangers on Earth." 
Si pour certains auteurs (Craddock 2001, Wesley Allen 2010), ce rituel religieux de participation produit un «dialogue» co-construit avec l'auditoire, il ne peut être 
assimilé ni au dialogue de l'oral conversationnel, ni aux phénomènes de dialogisme ou de "conversationnalisation", qui sont d'ailleurs absents dans le discours de Charleston. Un tel dialogue serait de même difficile à concilier avec la figure du surlocuteur. En effet, il s'agit d'une participation qui n'est possible que grâce à un locuteur qui assume, conformément à la Scène rhétorique, un statut de médiateur entre son auditoire et les valeurs qui fondent leur communauté. L'image du surlocuteur se double ici de celle du pasteur, figure paternelle pour la communauté, et médiateur entre la congrégation et Dieu.

La relation interpersonnelle qui se met en place et l'intégration de la communauté qui s'y opère sont tributaires du chant, et plus particulièrement du choix des paroles chantées : Amazing Grace. L'orateur s'en remet ici à l'autorité d'un " hyperénonciateur » à travers le processus de " particitation » (Maingueneau 2004), mot valise qui souligne la manière dont la citation de paroles connues et ancrées dans une culture témoigne de l'appartenance à une communauté qui se trouve renforcée par le discours. La citation est reconnue par le destinataire sans que l'on ait besoin de citer sa source. Il y a ainsi adhésion à un "Thésaurus » d'énoncés qui est «indissociable d'une communauté »: «Par son énonciation, le locuteur citant présuppose pragmatiquement que lui-même et son allocutaire sont membres de cette communauté » (2004:112). Parmi les formes de particitation figurent en bonne place, à côté des proverbes ou des contes populaires, les hymnes et les prières, qui relèvent d'une " particitation de communion » (ibid.; nous soulignons).

On constate une affinité naturelle entre particitation et Scène rhétorique : une dénivellation se creuse " entre la voix "ordinaire" du locuteur citant et une voix extraordinaire", celle d'un "hyperénonciateur», entité mythique qui garantit "son adéquation aux valeurs, aux fondements d'une collectivité » (ibid. 113) En l'occurrence ici, les paroles citées d'Amazing Grace, patrimoine de tous les Américains et de tous les Anglo-Saxons, sont identifiables non seulement par l'auditoire afro-américain, mais ainsi par l'ensemble de la communauté englobante.

Et ces paroles ne sont pas simplement énoncées, mais chantées, ce qui pousse au paroxysme la dimension sensorielle. La citation de paroles extraites d'un cantique fait partie intégrante du prêche afro-américain (on pense à la manière dont le discours de M. Luther King «I have a dream » se conclut par des vers du negro spirituel « Free at last »), avec passage parfois au mode chanté, y compris dans l'assemblée. Pour ceux qui écoutent, la musique les pénètre. Pour ceux qui choisissent de se joindre au chant, les voix et les corps ne font qu'un. Le chant renforce la focalisation sur le corps de l'orateur. Dans le cas du discours de Charleston, il s'agit d'une incorporation qui œuvre dans le sens de l'intégration au sein de la communauté. Ainsi fédérée, la communauté constituée de l'auditoire direct s'intègre plus facilement à la communauté englobante. Les autres membres de cette dernière, à savoir les Américains qui regardent la cérémonie (re)transmise, ne peuvent, à leur tour, qu'être touchés et ressentir ce sentiment d'appartenance.

51 Le premier mot chanté est précédé d'une pause de treize secondes qui, une fois n'est pas coutume, n'est pas remplie d'applaudissements.

16. If we can find that grace, / anything is possible. / If we can tap that grace, / everything can change/ [baisse de la voix]. Amazing grace / [pause: $4 \mathrm{sec}$.] Amazing grace [pause: $13 \mathrm{sec}$.$] .$ 

de communion qui s'ensuit : son regard devient plus déterminé ; le poing serré, son bras se baisse et se lève deux fois, ponctuant les parallélismes syntaxiques. Pendant le silence marqué qui précède le début du chant, Obama ne regarde pas son auditoire : il regarde vers sa droite, au-dessus des têtes, avant de diriger ses yeux vers le bas, vers le pupitre, comme s'il souhaitait s'isoler, se recentrer sur lui-même, pour marquer un moment d'introspection, avant le chant, qui ne dure que l'espace de quatre vers (v. plus haut l'ex. 12).

te, le Président reprend, telle une litanie, "sainteté impossible à circonscrire 》 (Rabatel 2015 : 34), la liste des noms des victimes. Ces derniers ne figurent plus dans des énoncés averbaux comme plus tôt dans le discours (v. plus haut l'ex. 11), mais s'intègrent cette fois dans des prédications répétées en position finale (épiphore), prenant pour complément « that grace » :

17. [en criant:] Clementa Pinckney found that grace. / Cynthia Hurd found that grace [audience: "yeah!"]. / Susie Jackson found that grace ["yeah!"]. / Ethel Lance found that grace [audience: "yeah!"]. / DePayne Middleton-Doctor found that grace ["yeah!"]. / Tywanza Sanders found that grace [“yeah!"]. [...]

Ces paroles sont martelées par une voix qui crie d'un débit rapide et qui crée un contraste avec les pauses ponctuant la fin de chaque énoncé, pauses remplies d'interjections de l'auditoire et des notes de l'organiste. Au final, il s'agit du point d'orgue du discours, moment de communion ultime visant à mobiliser orateur et membres de l'auditoire dans la même émotion, le même corps, pour un ultime hommage autour des noms des victimes. Le discours se clôt par une série d'anaphores ("May...May...») en guise de bénédiction, dont la pause marquée devant l'adjectif fortement accentué "United», prend acte de la cohésion nouvelle qui résulte de la communion et de l'intégration d'une communauté au sein d'une autre :

18. Through the example of their lives, / they've now passed it on to us. / May we find ourselves worthy of that precious / and extraordinary gift, / as long as our lives endure. / May grace now lead them home. / May God continue to shed His grace / on the United / States of America.

\section{Conclusion}

Nous avons tenté d'identifier différents procédés discursifs qui permettent de susciter la communion caractéristique de l'épidictique en prenant appui sur l'asymétrie constitutive de l'art oratoire. Cette communion joue ici sur deux niveaux en intégrant une communauté dans une autre, en mobilisant Scène rhétorique et particitation chantée. Le locuteur 'prend de la hauteur', devient surlocuteur, pour rassembler les destinataires dans une communauté et intégrer celle-ci dans une communauté englobante, le peuple américain. Le chant sollicite tout le corps et suscite une véritable incorporation. Il s'agit d'orchestrer un moment oratoire d'exception, à l'image de la grâce divine, objet d'émerveillement.

Ce discours se veut à l'image de l'homme que l'on commémore - à la fois sénateur et pasteur. Confronté au problème racial, Obama cherche à fondre le politique et le religieux pour les dépasser. A ce titre, le choix de cet hymne s'avère essentiel : écrit par un esclavagiste repenti, chanté par un Président lui-même métis qui achève son oraison funèbre en évoquant les "United States». Plutôt que de considérer comme une 
singularité cette fusion entre politique et religieux, on peut se demander si on ne retrouve pas là ce qu'il y a de commun entre ces deux champs: la construction d'un lien, la conversion d'une somme d'individus en une communauté qui transcende l'iciet-maintenant.

\section{BIBLIOGRAPHIE}

Alkebulan, Adisa. 2003. « The Spiritual Essence of African American Rhetoric », Jackson, Ronald \& Elaine Richardson (éds). Understanding African American Rhetoric: Classical Origins to Contemporary Innovations (New York: Routledge), 23-40

Amossy, Ruth. 2012. L'argumentation dans le discours (Paris : Colin)

Angermuller, Johannes. 2014. Poststructuralist Discourse Analysis, Subjectivity in Enunciative Pragmatics (New York: Palgrave Macmillan)

Atkinson, Maxwell. 1984 « Public Speaking and audience responses », Atkinson, Maxwell \& John Heritage (éds). Structures of Social Action (Cambridge: Cambridge University Press), 370-409

Bakhtine, Mikhail. 1984. Esthétique de la création verbale (Paris : Gallimard)

Benoït à la Guillaume, Luc. 2012. Quand la Maison-Blanche prend la parole : le discours présidentiel de Nixon à Obama (Bern : P. Lang)

Berry, Mary Frances \& Josh Gottheimer. 2010. Power in Words: The Stories Behind Barack Obama's Speeches, from the State House to the White House (Boston: Beacon Press)

Calloway-Thomas, Carolyn, Lucaites, John (éds). 1993. Martin Luther King Jr. and the Sermonic Power of Public Discourse (Tuscaloosa: University of Alabama Press)

Capone, Alessandro. 2010. « Barack Obama's South Carolina Speech », Journal of Pragmatics 42, 2964-2977

Craddock, Fred. 2001. As One Without Authority. (St Louis, Missouri: Chalice Press)

Crystal, David. 2001. Language and the Internet (Cambridge: Cambridge University Press)

Debras, Camille \& Emilie L'Hôte. 2015. « Framing, metaphor and dialogue: a multimodal approach to party conference speeches ", Metaphor and the Social World 5 (2), 184-204

Debras, Camille \& Fiona Rossette (à paraître) « Now is the time'; the Interaction of Prosody and Syntax in Martin Luther King's Speech 'I have a dream' ».

Dominicy, Marc \& Madeleine Frédéric (éds). 2001. La mise en scène des valeurs : la rhétorique de l'éloge et du blâme (Lausanne : Delachaux et Niestlé)

Duez, Danielle. 2003. « Le pouvoir du silence et le silence du pouvoir : comment interpréter le discours politique ", MediaMorphoses 8, 77-82

Engelke, Matthew. 2004. « Text and performance in an African church: The book, 'live and direct' », American Ethnologist 31, 76-91 
Fairclough, Norman. 1994. «Conversationalisation of public discourse and the authority of the consumer ", Keat, Russell, Nigel Whitely \& Nicholas Abercrombie (éds). The Authority of the consumer (London: Routledge), 235-249

Ferrera, Mark. 2013. Barack Obama and the Rhetoric of Hope (Jefferson, North Carolina, London: McFarland \& Company)

Goffman, Erving. 1981. Forms of Talk (Philadelphia: University of Pennsylvania Press)

Heritage, John \& David Greatbatch. 1986. « Generating Applause: A Study of Rhetoric and Response at Party Political Conferences ", American Journal of Sociology 92 (1), 110-157

Harrison Robert \& , Linda Harrison. 1993. « The Call from the Mountaintop: Call-Response and the Oratory of Martin Luther King, Jr. ", Calloway-Thomas, Carolyn \& John Lucaites (éds). Martin Luther King Jr. and the Sermonic Power of Public Discourse, (Tuscaloosa: University of Alabama Press), $162-178$

Horton, Donald \& Richard Wohl. 1956. « Mass communication and para-social interaction: Observations on intimacy at a distance », Psychiatry 19, 215-229

Koren, Roseline. 2015. «Une instance à la croisée du discours et de l'éthique : le « surdestinataire », Angermuller, Johannes \& Gilles Philippe (éds). Analyse du discours et dispositifs d'énonciation. Autour des travaux de Dominique Maingueneau (Limoges : Lambert-Lucas), 137-146

Lischer, Richard. 1995. The Preacher King: Martin Luther King, Jr. and the Word that Moved America (Oxford: Oxford University Press)

Magri-Mourgues, Véronique. 2015. «L'anaphore rhétorique dans le discours politique. L'exemple de N. Sarkozy », Semen 38, version en ligne, consultée le 11/1/2016, https://semen.revues.org/ 10319

Maingueneau, Dominique. 2004. « Hyperénonciateur et particitation », Langages 156, 111-126

Maingueneau, Dominique. 2014. Discours et analyse du discours. (Paris : Colin)

Maingueneau, Dominique. 2016. « Apostrophe et Scène rhétorique », Biglari, Amir, Geneviève Salvan (éds). Figures en discours (Louvain-la-Neuve : Academia-l'Harmattan)

Malmström, Hans. 2016. « Engaging the Congregation: The Place of Metadiscourse in Contemporary Preaching », Applied Linguistics 37-4. 561-582

O. Wesley, Allen. 2010. The Renewed Homiletic (Minneapolis: Augsburg Fortress Publishers)

Prasch, Alison. 2015. « Reagan at Pointe du Hoc: Deictic Epideictic and the persuasive power of "bringing before the eyes" ", Rhetoric and Public Affairs 18 (2), 247-276

Perelman, Chaim \& Lucie Olbrecht-Tyteca. 1970 [1958]. Traité de l'argumentation. La nouvelle rhétorique (Bruxelles : Editions de l'Université de Bruxelles)

Queen, Robin. 1992. "Prosodic organization in the speeches of Martin Luther King ", Proceedings of the IRCS workshop on prosody in natural speech, Philadelphia, 151-160

Rabatel, Alain. 2015. « Des répétitions dans le discours religieux : l'exemple des litanies », Le discours et la langue 7-2 23-39

Rosenberg, Bruce. 1971. Can These Bones Live? The Art of the American Folk Preacher (Oxford: Oxford University Press)

Rossette, Fiona. 2017a. Prendre la parole en anglais (Paris : Colin) 
Rossette, Fiona. 2017b. « Discursive divides and Rhetorical Staging, or the transcending Function of Oratory ", Journal of Pragmatics 108, 48-59

Shih, Chilin \& Greg Kochanski. 2001. « Prosody Control for Speaking and Singing Styles », Eurospeech Proceedings, 669-672

Ure, Jean. 1971. « Lexical density and register differentiation », Perren, George \& John Trim (éds). Applications of Linguistics: Selected Papers of the Second International Congress of Applied Linguistics, Cambridge 1969 (Cambridge: Cambridge University Press), 443-452

Wills, Garry. 1992. Lincoln at Gettysburg. The Words that Remade America (New York: Simon \& Schuster)

Wilson, John. 2015. Talking with the President. The Pragmatics of Presidential Language (Oxford: Oxford University Press)

\section{NOTES}

1. «Obama's Eulogy, Which Found Its Place in History », New York Times 3/7/2015, consulté le $22 / 10 / 2016$.

2. La spécificité de l'épidictique tient au fait qu'il « se propose d'accroître l'intensité de l'adhésion à certaines valeurs reconnues par l'auditoire [...] en se servant de l'ensemble des moyens dont dispose la rhétorique pour amplifier et valoriser » (Perelman et Olbrechts-Tyteca $(1970: 67$; nous soulignons).

3. "[A]n address somewhere between a presidential speech and a sermon", Sam Leith, The Financial Times 6/7/2015

4. Publié en 1779, il fut écrit par John Newton, à la fois clergyman anglais et esclavagiste repenti.

5. Les termes de «surlocuteur » et d'« hyperénonciateur » qui s'appliquent tous deux au locuteur du discours de Charleston recouvrent deux réalités distinctes : contrairement au surlocuteur, terme qui désigne le rôle d'un participant au sein de l'échange, l'hyperénonciateur correspond à un être en quelque sorte mythique qui existe comme support plus abstrait d'une énonciation - à l'instar du support énonciatif qui motive la citation de proverbes au sein d'une culture donnée.

6. Hormis le domaine politique, un tel dialogisme, ou effet de conversationnalisation, caractérisent les nouvelles formes de prise de parole en public qui se sont développées ces deux dernières décennies d'après le modèle de la communication à l'anglo-saxonne (ex. TED talks, keynotes, sales pitch, voir Rossette, 2017a).

7. Chez Maingueneau, le concept de Scène rhétorique n'est pas assimilable à l'un des trois composants de sa scène d'énonciation. En effet, alors que les concepts de scène générique, scène englobante et scénographie tentent de saisir la tension entre régularités et écarts à l'intérieur des genres discursifs, la scène rhétorique correspond à un dispositif transverse aux genres, qui caractérise des instances non seulement d'art oratoire mais également d'autres contextes discursifs, comme la poésie. Cela explique sans doute le choix du S majuscule pour « Scène ", que j'ai choisi de conserver ici.

8. Le concept de surdestinataire est à distinguer de celui d' "auditoire composite ", terme employé par Perelman et Olbrecht-Tyteca (1969) pour souligner la nécessité de mobiliser différents types d'arguments pour toucher un auditoire composé d'individus différents les uns des autres. Pour une présentation plus détaillée du concept de surdestinataire associé à l'élévation, voir R. Koren (2015).

9. L'emploi du diminutif «Clem » s'avère également stratégique pour souligner qu'il adopte le point de vue de la communauté noire - alors qu'Obama déclare, au début de son discours, qu'il ne 
connaissait pas le pasteur à titre personnel (« I cannot claim to have the good fortune to know Reverend Pickney well »).

10. La vidéo du discours de Charleston peut être visionnée au lien suivant: https:// www.youtube.com/watch?v=x9IGyidtfGI, et celle de Tucson au lien https://www.youtube.com/ watch?v=bXd4-9AkWL0.

11. Comme le souligne Amossy (2012), la nouvelle rhétorique de Perelman «réhabilite» l'épidictique, alors que la rhétorique classique « voyait dans ses morceaux d'apparat un spectacle plutôt qu'une pratique oratoire tendue vers un but précis » (Amossy 2012 :19).

12. Selon l'ordre d'apparition dans le discours : "A man who... » (3 occurrences); «A place...» (2) ; « When there... » (2) ; «An act...» $(2)$; «The grace... » (3) ; «It would be... » (2) ; «Perhaps it... » (2) ; « May... » (3).

13. Comme l'explique Rabatel (2015:36), le discours religieux « entretient un rapport particulier avec la répétition », notamment par «l'importance accordée à l'évocation de l'absent ». Un tel effet est exemplifié par la litanie, qui participe à l'effet de communion puisqu'elle vise «un accomplissement de soi qui cherche aussi à faire advenir la communauté dans le partage et les harmoniques d'une parole commune, une et pluriel ».

14. A titre de comparaison, l'énoncé averbal est absent de l'éloge de Tucson, qui comporte par ailleurs moins de figures.

15. Cf. les expressions en anglais "stand up for what you believe in"; "stand up and be counted».

\section{RÉSUMÉS}

L'éloge funèbre prononcé par Barack Obama en mémoire de Clementa Pinckney, le pasteur noir assassiné dans son église à Charleston, fut très commenté dans les médias pour ce qui y a tenu lieu de péroraison : le Président a entonné l'hymne religieux le plus célèbre de la culture anglosaxonne, Amazing Grace. Dans cette contribution, je m'attache à montrer comment la parole vise à susciter le sentiment de communion qui caractérise le genre épidictique. Il s'agit de montrer comment Obama parvient à intégrer l'auditoire direct, physiquement présent dans la salle, à savoir la communauté afro-américaine, au sein d'une communauté englobante, c'est-à-dire l'ensemble du peuple américain. Pour cela je m'appuie sur les concepts de "Scène rhétorique " (Maingueneau 2016) et de "particitation» (Maingueneau 2004). Cette étude met en avant la position particulière d'un orateur qui "prend de la hauteur ». En même temps, les différentes formes de participation de l'auditoire, qui proviennent du rituel religieux afro-américain, et dont le chant représente le point culminant, jouent un rôle crucial pour susciter la communion. Celleci repose également sur la mise en spectacle de la parole, à travers le déploiement de figures rhétoriques, les variations vocales et rythmiques, et la gestuelle. Ces procédés, amplifiés encore une fois par le chant, engagent tout le corps et concourent à une véritable incorporation qui suscite l'intégration au sein de la communauté englobante.

The eulogy delivered by Barack Obama in homage to Clementa Pinckney, the black pastor who was assassinated in his church in Charleston, triggered much commentary in the media, due to the peroration, when Obama actually broke out into song, singing several verses from the most famous hymn of the English language, Amazing Grace. In this study, I examine the ways in which the speech generates the sense of communion that is so central to epideictic oratory. I 
demonstrate how Obama succeeds, from a discursive point of view, in integrating his live audience (the Black American community) within the overarching community of the American people. In order to do so, I draw on the concepts of "Rhetorical Staging" (Maingueneau 2016) and "particitation" (Maingueneau 2004). I examine the specific status of the orator, who "rises above" his/her audience, precisely in order to bring different components of the audience together within the same community. The different forms of participation by the audience, which are part of Afro-American religious ritual, play a crucial role in bringing about the communion. This is also achieved via a staging of the word, rhetorical figures, vocal and rhythmic variation, and body language. These phenomena culminate in the song, and involve the whole body. Such an embodiment plays a major role in the integration of the audience into the overarching community.

INDEX

Keywords : communion, epidictic oratory, particitation, rhetorical staging, superspeaker

Mots-clés : communion, épidictique, particitation, Scène rhétorique, surlocuteur

\section{AUTEUR}

\section{FIONA ROSSETTE}

Université Paris Nanterre 